\title{
Effect of acute football activity and physical fitness on glycaemic and insulinaemic responses in adolescents
}

Ryan A. Williams, Simon Cooper, Karah J. Dring , Lorna Hatch , John G. Morris, Caroline Sunderland \& Mary E. Nevill

To cite this article: Ryan A. Williams, Simon Cooper , Karah J. Dring , Lorna Hatch , John G. Morris , Caroline Sunderland \& Mary E. Nevill (2021): Effect of acute football activity and physical fitness on glycaemic and insulinaemic responses in adolescents, Journal of Sports Sciences, DOI: 10.1080/02640414.2020.1860362

To link to this article: https://doi.org/10.1080/02640414.2020.1860362

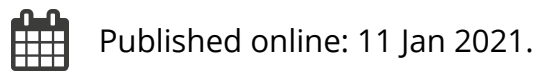

Submit your article to this journal ๘

山ll Article views: 21

Q View related articles ¿

View Crossmark data ¿` 


\title{
Effect of acute football activity and physical fitness on glycaemic and insulinaemic responses in adolescents
}

\author{
Ryan A. Williams $\mathbb{D}$, Simon Cooper $\mathbb{D}$, Karah J. Dring $\mathbb{D}$, Lorna Hatch, John G. Morris $\mathbb{D}$, Caroline Sunderland $\mathbb{D}$ \\ and Mary E. Nevill (D)
}

Exercise and Health Research Group; Sport, Health and Performance Enhancement (SHAPE) Research Centre; Department of Sport Science; Nottingham Trent University, Nottingham, UK

\begin{abstract}
The present study examined the metabolic responses to an acute bout of football and the overall and moderating role of physical fitness on these responses, in adolescents. Thirty-six adolescents (16 girls, 20 boys; $12.6 \pm 0.5 \mathrm{y})$ completed two trials $(60-\mathrm{min}$ football and $60-\mathrm{min}$ seated rest) separated by 7-d. Capillary blood samples were taken at baseline (60-min prior to exercise/rest), immediately, 30and 60-min post-exercise and 30-, 60- and 120-min following a standardised lunch (1.5-, 2- and 3-h postexercise), for the determination of blood glucose and plasma insulin concentrations. The median split of distance covered on the multi-stage fitness test was used to define high- and low-fit groups. Overall plasma insulin tAUC following lunch was lower in high-fit participants compared to low-fit (high-fit: $3784.2 \pm 1653.1 \mathrm{pmol} \cdot \mathrm{L}^{-1} \times 120 \mathrm{~min}$, low-fit: $\left.6457.3 \pm 3290.7 \mathrm{pmol} \cdot \mathrm{L}^{-1} \times 120 \mathrm{~min} ; p<0.001\right)$, although there was no acute effect of the football session $(p>0.05)$. Football reduced blood glucose concentration 1-h postexercise compared to control (exercise: $3.8 \pm 0.6 \mathrm{mmol} \cdot \mathrm{L}^{-1}$, rest: $4.6 \pm 0.8 \mathrm{mmol} \cdot \mathrm{L}^{-1} ; p<0.001$ ), but this was similar for the high- and low-fit participants $(p>0.05)$. Blood glucose tAUC was not affected by exercise or physical fitness ( $p>0.05)$. These data emphasise the importance of physical fitness for metabolic health in adolescents, as well as the utility of football as a popular form of games-based activity for improving glucose regulation.
\end{abstract}

ARTICLE HISTORY

Accepted 3 December 2020

KEYWORDS

Football; metabolic health; adolescents; acute exercise; postprandial response

\section{Introduction}

Physical inactivity is associated with poor cardiometabolic health during adolescence (Ekelund et al., 2007, 2012) and is considered an important risk factor in the development of most chronic diseases (Booth et al., 2012). It is becoming a highly pertinent issue, highlighted by the increasing prevalence of type 2 diabetes amongst adolescents (Tagi et al., 2019). Insulin resistance, and reduced glucose tolerance, are involved in the aetiology of type 2 diabetes (Reaven, 2005); which presents them as targets to deal with the increasing prevalence of type 2 diabetes in this population and to enhance lifelong health. The postprandial response to a test meal, particularly the insulinaemic response, is gaining recognition as a key risk factor for the development of insulin resistance and, ultimately, cardiometabolic disease (DiNicolantonio et al., 2017; Lautt, 2007). There are numerous risk factors associated with the development insulin resistance, some of which can be modified through lifestyle behaviour change (Tuomilehto et al., 2001), such as body composition (particularly central adiposity) (Arslanian and Suprasongsin, 1996; Arslanian, 2000) and physical inactivity (Reinehr, 2013). Of these risk factors, physical activity offers an attractive target for public health initiatives, with participation in physical activity a recommended and cost-effective method to combat the development of metabolic risk factors in young people (Gleeson et al., 2011; Röhling et al., 2016). Indeed, such public health initiatives are needed as it is estimated that only $24 \%$ of boys and $18 \%$ of girls (aged 5 to $15 \mathrm{y}$ ) meet the recommended guidelines of 60 min moderate-vigorous physical activity per day (Scholes \& Mindell, 2016).

High-intensity intermittent exercise is an increasingly popular method for implementing physical activity in young people due to the associated health benefits and greater perceived enjoyment (Batacan et al., 2017; Bond et al., 2017). Recent laboratory-based research in adolescents has focused on high-intensity intermittent exercise, using $8 \times 1 \mathrm{~min}$ bouts at $90 \%$ of peak power on a cycle ergometer (Cockcroft et al., 2015, 2017). These findings demonstrate transient improvements in the postprandial insulinaemic and glycaemic response to an oral glucose tolerance test (Cockcroft et al., 2015, 2017), which has important implications for lifelong metabolic health, given their role as a known risk factor for the development of cardiometabolic diseases (DiNicolantonio et al., 2017; Lautt, 2007). Whilst these findings are promising, it could be argued that the modality of exercise, as well as the glucose tolerance test (rather than a test meal), are not ecologically valid. Interestingly, $85 \%$ of young people in the UK who met the recommended daily target of 60 minutes moderate-vigorous physical activity do so through informal sports (Townsend et al., 2015). It is worth noting that the most popular sport amongst British adolescents is football, with $45 \%$ of $11-15$ year olds participating in football. The activity patterns seen in team sports like football are known to be intermittent (Svensson \& Drust, 2005), much like the natural activity patterns of adolescents (Bailey et al. 
1995; Howe et al., 2010). It has thus been suggested that smallsided games could be beneficial for health promotion (Krustrup \& Bangsbo, 2015), with empirical evidence demonstrating that acute small-sided games (Rugby Union) improve metabolic health in sedentary adult men (Mendham et al., 2012, 2015). However, there is little research investigating the effects of team games on risk factors for cardiometabolic disease in adolescents.

Smallcombe et al., (2018) employed a $48 \mathrm{~min}$ bout of football (using small-sided games) compared to duration-matched treadmill running in adolescent boys (12.7-13.3 y). The bout of football led to greater improvements in postprandial lipaemia in adolescent boys - despite less distance being covered in the small-sided games. However, to the authors' knowledge, only one study has examined the effects of games-based exercise on postprandial glycaemia and insulinaemia in adolescents (Dring et al., 2019a). A 60 min bout of basketball, including skill drills and small-sided games, led to a $35 \%$ reduction in the postprandial insulinaemic response to a standardised mixed-meal (Dring et al., 2019a); which is consistent with laboratory findings utilising high-intensity intermittent cycling protocols (Cockcroft et al., 2015). However, it is unknown how an acute bout of football may affect the glycaemic and insulinaemic responses to a standardised mixed-meal, in adolescents. This is important gap to fill, given the popularity and ecological validity of football.

Physical fitness is also strongly associated with metabolic health in adolescents. Specifically, higher fit adolescents have a lower degree of fasting insulin resistance, compared to their low-fit counterparts (Dring et al., 2019b; Haapala et al., 2020). Despite this, it is unknown if the physical fitness of participants moderates the acute metabolic responses to exercise and whether there are any overall associations of fitness with postprandial insulinaemia and glycaemia. It has been suggested that those considered lower fit will see greater metabolic improvements from acute exercise than their higher fit counterparts (Cockcroft et al., 2015; Short et al., 2018, 2013). On the other hand, it has also been suggested that those with a higher physical fitness will benefit more, by virtue of the ability to work at a higher absolute exercise intensity (Dring et al., 2019a). However, these suggestions have been made comparing across studies, with different methods of assessment for physical fitness. Thus, no empirical study to date has directly investigated the moderating role of physical fitness on the metabolic responses following an acute bout of exercise.
Therefore, the aims of the current study were to examine: i) the acute effects of football activity on the postprandial glycaemic and insulinaemic responses to a standardised meal; ii) to compare the postprandial responses between high- and low-fit adolescents; and iii) how physical fitness may moderate the acute effects of exercise on these postprandial responses. It was hypothesised that the acute bout of football would reduce the postprandial glycaemic and insulinaemic response. It was also hypothesised that low fit adolescents would have greater glycaemic and insulinaemic responses compared to high-fit adolescents, with the acute reduction following football activity being greater in the low-fit participants.

\section{Methods}

\subsection{Participant characteristics}

Thirty-six adolescents (20 boys, 16 girls; 12-13 years old) volunteered to participate in the study. During familiarisation, all participants underwent anthropometric measurements consisting of height $(\mathrm{cm})$, body mass $(\mathrm{kg})$ and sitting height (cm). These were used to calculate age at peak height velocity, which has been described previously (Moore et al., 2015), as it provides a non-invasive measure of maturity offset as a descriptive characteristic of participants' maturational status. Height was measured with a Leicester Height Measure (Seca, Hamburg, Germany) accurate to $0.1 \mathrm{~cm}$ and body mass was measured using a Seca 770 digital scale (Seco, Hamburg, Germany) accurate to $0.1 \mathrm{~kg}$. Waist circumference was measured at the narrowest abdominal point, between the lower margin of the lowest palpable rib and the iliac crest, to the nearest $0.1 \mathrm{~cm}$ (World Health Organisation, 2008). Four skinfold sites were measured (tricep, subscapular, supraspinale and front thigh) as a surrogate of body composition, in line with previously described methods (Dring et al., 2019a). Descriptive participant characteristics are presented in Table 1.

\subsection{Experimental design}

The study conformed to the Declaration of Helsinki guidelines and was approved by the Nottingham Trent University Human Ethics Committee. Participants were recruited from local secondary schools in the East Midlands area of the UK. Written

Table 1. Participant characteristics for the group overall, as well as for the high- and low-fit groups. Data are mean \pm SD.

\begin{tabular}{|c|c|c|c|c|}
\hline Variable & $\begin{array}{c}\text { Overall } \\
(\mathrm{n}=36 ; \\
16 \text { girls, } 20 \text { boys })\end{array}$ & $\begin{array}{c}\text { High-fit } \\
(\mathrm{n}=18 ; \\
8 \text { girls, } 10 \text { boys })\end{array}$ & $\begin{array}{l}\text { Low-fit }(\mathrm{n}=18 ; \\
8 \text { girls, } 10 \text { boys) }\end{array}$ & Independent samples t-test $(p)$ \\
\hline Age (yrs) & $12.6 \pm 0.5$ & $12.7 \pm 0.5$ & $12.4 \pm 0.5$ & - \\
\hline Height $(\mathrm{cm})$ & $163.1 \pm 7.0$ & $163.5 \pm 8.0$ & $162.6 \pm 6.1$ & 0.709 \\
\hline Body Mass (kg) & $53.9 \pm 10.0$ & $50.1 \pm 8.9$ & $57.6 \pm 10.0$ & 0.024 \\
\hline$B M I\left(\mathrm{~kg} \cdot \mathrm{m}^{-2}\right)$ & $20.2 \pm 3.0$ & $18.6 \pm 2.0$ & $25.7 \pm 3.1$ & 0.001 \\
\hline BMI Percentile & $63.1 \pm 30.0$ & $48.9 \pm 26.9$ & $77.3 \pm 26.6$ & 0.003 \\
\hline Waist Circumference $(\mathrm{cm})$ & $70.0 \pm 8.0$ & $66.0 \pm 5.4$ & $73.9 \pm 8.4$ & 0.002 \\
\hline Sum of 4 skinfolds ( $\mathrm{mm}$ ) & $60.3 \pm 26.8$ & $42.8 \pm 12.8$ & $77.8 \pm 25.7$ & $<0.001$ \\
\hline Maturity Offset & $0.08 \pm 0.94$ & $0.20 \pm 0.84$ & $-0.05 \pm 1.03$ & 0.449 \\
\hline MSFT Distance $(m)$ & $1160 \pm 400$ & $1480 \pm 300$ & $840 \pm 140$ & $<0.001$ \\
\hline Predicted $\dot{\mathrm{V}} \mathrm{O}_{2}$ peak $\left(\mathrm{ml} \cdot \mathrm{kg}^{-1} \cdot \mathrm{min}^{-1}\right)^{a}$ & $47.9 \pm 5.2$ & $52.0 \pm 3.7$ & $43.8 \pm 2.5$ & $<0.001$ \\
\hline
\end{tabular}

Abbreviations: MSFT = Multi-Stage Fitness Test.

apredicted from the multi-stage fitness test using the equations of Barnett et al. (Barnett et al., 1993) 
parental consent and child assent were obtained during the initial phase of recruitment. A health screen was completed by the participants' parent/guardian and was checked by a lead investigator to ensure there were no medical conditions that would affect the child's participation.

The study employed a randomised, order-balanced, crossover, within-subjects design with two main experimental trials (exercise and resting), separated by at least $7 \mathrm{~d}$. Participants were blind to the trial condition until arrival at school. A familiarisation took place $7 \mathrm{~d}$ before the first main trial and allowed participants to be acquainted with all the necessary procedures, including capillary blood sampling and a battery of cognitive function tests (data published separately; Williams et al., under review). Participants were also familiarised with a football session, consisting of skill drills and small-sided games.

During the familiarisation participants also completed the multi-stage fitness test (MSFT) (Ramsbottom et al., 1988) for the assessment of physical fitness. Prior to the start of the MSFT, participants were fitted with a heart rate monitor (Firstbeat Team Sport System, Firstbeat Technologies Ltd, Finland). Heart rate was monitored throughout the MSFT and maximum heart rate was recorded upon completion. Participants were paced by a member of the research team who run alongside them and to encourage maximum effort from the participants, verbal encouragement was provided. Performance on the test was determined by the total distance covered $(\mathrm{m})$, with participants assigned to high- and low-fitness groups, based on sexspecific median splits of MSFT distance covered. This resulted in 18 low-fit participants ( 8 girls, 10 boys) and 18 high-fit participants ( 8 girls, 10 boys). This criterion was used based on previous work demonstrating that the MSFT is a good indicator of cardiometabolic health in adolescents (Dring et al., 2019b) and it is a valid (Tomkinson et al., 2019) and reliable measure of endurance capacity in adolescents (Artero et al., 2011).

\subsubsection{Main trials}

Participants were instructed to record their dietary intake for the 24 hours preceding the first experimental trial and during evening one of the first main trial. Recorded diets were then replicated for the second main trial. Participants were asked to refrain from eating or drinking from $9 \mathrm{pm}$ prior to the two main trials. Water was allowed ad libitum at all times. Participants were also asked to refrain from physical activity $24 \mathrm{~h}$ prior to the main trials and were not permitted to participate in any strenuous physical activity until the final measurement had been taken on the second day of an experimental trial. Parents/ guardians were contacted by telephone in the evening prior to each main trial to ensure compliance with these requirements.

On the morning of the main trials, participants reported to school at the beginning of the usual school day (between 8 am and 8:30 am). Upon arrival participants had a fasted capillary blood sample taken and were fitted with a heart rate monitor (Firstbeat Team Sport System, Firstbeat Technologies Ltd, Finland), to be worn throughout the trial. Participants were then given a standardised breakfast and $1 \mathrm{~h}$ after the start of breakfast, they either took part in a 60 min exercise session (Football; see Section 2.3.3) or remained quietly seated in the classroom. Capillary blood samples were taken immediately, 30 and $60 \mathrm{~min}$ following the exercise/rest period. Lunch was provided, with additional blood samples at 30,60 and $120 \mathrm{~min}$ post-lunch (1.5, 2 and $3 \mathrm{~h}$ post-exercise). Participants returned at the same time on the following morning (Day 2) for an additional fasted capillary blood sample. Both trials followed a time-matched protocol (lasting $\sim 5.5 \mathrm{~h}$ ), with the only difference being the $60 \mathrm{~min}$ exercise session. A schematic of the experimental protocol can be seen in Figure 1.

\subsection{Experimental procedures}

\subsubsection{Standardised breakfast and lunch}

A standardised breakfast and lunch were provided for participants during the experimental trials. The breakfast provided $1.5 \mathrm{~g} \cdot \mathrm{kg}^{-1}$ body mass of carbohydrate (cornflakes, milk, white toast and butter). The lunch also provided $1.5 \mathrm{~g} \cdot \mathrm{kg}^{-1}$ body mass of carbohydrate (chicken sandwich, baked crisps and an apple; with a cheese alternative for vegetarians); which was provided $1 \mathrm{~h}$ post-exercise. Participants were given $15 \mathrm{~min}$ to consume breakfast and lunch. Both meals have been used in previous research examining postprandial responses (Cooper et al., 2016; Dring et al., 2019a) in a similar study population.

\subsubsection{Capillary blood samples}

In order to increase capillary blood flow, participants' hands were warmed via submersion in warm water. A single-use lancet (Unistik Extra, 21 gauge, $2.0 \mathrm{~mm}$ depth; Owen Mumford Ltd, Oxford, UK) was used and the blood was collected into two $300 \mu \mathrm{l}$ EDTA coated microvettes (Sarstedt Ltd, UK). A single $25 \mu \mathrm{l}$ whole blood sample was also collected, using a pre-calibrated glass pipette (Hawksley Ltd, UK), and immediately deproteinised in $250 \mu$ ice-cooled $2.5 \%$ perchloric

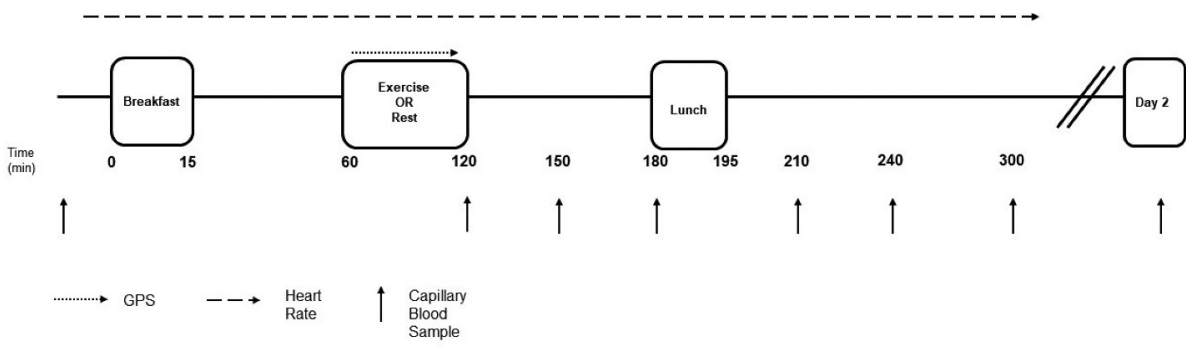

Figure 1. Visual representation of the experimental trials. The timeline shown is in cumulative minutes. Vertical arrows denote timing of blood samples, whilst the horizontal arrows represent the duration of heart rate monitoring and GPS tracking 
acid. Samples were centrifuged at $1000 \times \mathrm{g}$ for $4 \mathrm{~min}$ at $4^{\circ} \mathrm{C}$ (Eppendorph 5415 C, Hamburg, Germany). Plasma was removed from the microvettes and placed into $250 \mu$ plastic vials for subsequent analysis. All samples were frozen immediately at $-20^{\circ} \mathrm{C}$ and transferred to $\mathrm{a}-80^{\circ} \mathrm{C}$ freezer as soon as possible.

Blood glucose concentrations were determined, in duplicate, using a commercially available assay (GOD/PAP method, GL364, Randox, Ireland). Plasma insulin concentrations were determined using a commercially available ELISA (Mercodia Ltd, Sweden). The intra-assay coefficient of variation (CV) of the assays, based on eight repeat measurements, was $3.7 \%$ for blood glucose concentration and $4.8 \%$ for plasma insulin concentration. Blood glucose and plasma insulin total area under the curve (tAUC) following the standardised lunch were calculated (GraphPad Prism 7, GraphPad Software, USA), using methods described previously (Wolever et al., 1991).

\subsubsection{Exercise protocol}

The exercise consisted of a 60 min football session. Football was chosen as it intermittent in nature, which replicates the natural activity patterns of adolescents (Bailey et al., 1995), as well as being an enjoyable and popular form of games-based activity for young people. An experienced football coach delivered the sessions to groups of 10 participants, on outdoor facilities at the respective schools. The session consisted of a warm-up (jogging around the pitch and sprinting between cones; 5 min), skill-based drills (passing, dribbling and ball control; $25 \mathrm{~min}$ ) and small-sided games (5 vs 5, 4 min games with 1 min break to switch teams; $30 \mathrm{~min}$ ). At the end of the session, participants walked from the outdoor pitch to the classroom, which acted as a brief cool-down. Heart rate was monitored throughout the session. Maximum heart rate (HRmax), as recorded at the end of the MSFT, and heart rate during the football session were used to calculate the relative exercise intensity (\%HRmax). Additionally, Global Positioning System (GPS) devices were worn to quantify the external load during the football session using SPI HPU (15 Hz) portable GPS units (GPSports, Australia). The GPS units were fitted to sit between the scapulae, at the base of the cervical spine, using an elasticated shoulder harness. After each exercise session, the data were downloaded to
Team AMS software (Team AMS, GPSports). The variables of interest were total distance covered $(\mathrm{m})$ as well as the distance covered in six different speed categories: standing $(\leq 0.4 \mathrm{~km} \cdot \mathrm{h}-$ ${ }^{-1}$ ), walking ( $>0.4$ to $3.0 \mathrm{~km} \cdot \mathrm{h}^{-1}$ ), low-intensity running $(>3.0$ to $\left.8.0 \mathrm{~km} \cdot \mathrm{h}^{-1}\right)$, moderate-intensity running $\left(>8.0\right.$ to $\left.13.0 \mathrm{~km} \cdot \mathrm{h}^{-1}\right)$, high-intensity running (>13.0 to $18.0 \mathrm{~km} \cdot \mathrm{h}^{-1}$ ) and sprinting $\left(>18.0 \mathrm{~km} \cdot \mathrm{h}^{-1}\right.$ ) (Smallcombe et al., 2018).

\subsection{Statistical analysis}

All data analysis was conducted using SPSS (Version 24, SPSS Inc, Chicago, IL, USA). Data were assessed for normality using the Shapiro-Wilk test and visual inspection of histograms and Q-Q plots, which revealed that all data (except from insulin) were normally distributed. Insulin data were log-transformed prior to analysis. Heart rate during the 60 min football session was compared between the high- and low-fit groups using an independent samples t-test. The distance covered across the six speed categories during the football session were analysed between the high- and low-fit group via multivariate analysis of variance (MANOVA). Blood glucose and plasma insulin concentration were analysed via three-way (trial*time*fitness) mixed model analysis of variance (ANOVA), with repeated measures for trial and time, and fitness as a between-subjects factor. Blood glucose and plasma insulin tAUC were analysed using a two-way (trial*fitness) mixed-model ANOVA, with repeated measures for trial and fitness as a between-subjects factor. Where main effects between fitness groups and trials existed, the mean difference (MD) and the 95\% confidence interval (Cl) are presented. Data are presented as mean $\pm \mathrm{SD}$, unless otherwise stated. For all analyses, statistical significance was accepted as $p<0.05$.

\section{Results}

\subsection{Exercise characteristics}

During the $60 \mathrm{~min}$ football session, average heart rate was $151 \pm 16$ beats min $^{-1}$, maximum heart rate was $186 \pm 13$ beats $\min ^{-1}$ and relative exercise intensity was $75 \pm 8 \%$ HRmax (Table 2). Average $\left(t_{(32)}=-2.8, p=0.009\right)$ and maximum

Table 2. Average and maximum heart rate, relative exercise intensity and GPS characteristics for the group overall, as well as the high- and low-fitness splits, during the 60 min Football session. Data are mean \pm SD.

\begin{tabular}{|c|c|c|c|}
\hline Variable & $\begin{array}{c}\text { Overall } \\
(\mathrm{n}=36 \\
16 \text { girls, } 20 \text { boys })\end{array}$ & $\begin{array}{c}\text { High-fit } \\
(\mathrm{n}=18 ; \\
8 \text { girls, } 10 \text { boys })\end{array}$ & $\begin{array}{l}\text { Low-fit }(\mathrm{n}=18 \\
8 \text { girls, } 10 \text { boys) }\end{array}$ \\
\hline \multicolumn{4}{|l|}{ Heart Rate } \\
\hline Average Heart Rate (beats·min ${ }^{-1}$ ) & $151 \pm 16$ & $144 \pm 16$ & $158 \pm 12^{*}$ \\
\hline Maximum Heart Rate (beats. $\mathrm{min}^{-1}$ ) & $186 \pm 13$ & $180 \pm 11$ & $191 \pm 11^{*}$ \\
\hline $\begin{array}{l}\text { Relative Exercise Intensity } \\
\quad(\% \text { maximum heart rate })^{b}\end{array}$ & $75 \pm 8$ & $70 \pm 7$ & $80 \pm 6^{*}$ \\
\hline \multicolumn{4}{|l|}{ Distance covered $(m)$ within speed categories } \\
\hline Standing $\left(\leq 0.4 \mathrm{~km} \cdot \mathrm{h}^{-1}\right)$ & $6 \pm 2$ & $7 \pm 1$ & $6 \pm 2$ \\
\hline Walking $\left(>0.4\right.$ to $\left.3.0 \mathrm{~km} \cdot \mathrm{h}^{-1}\right)$ & $419 \pm 64$ & $398 \pm 48$ & $439 \pm 72$ \\
\hline Low-intensity running $\left(>3.0\right.$ to $\left.8.0 \mathrm{~km} \cdot \mathrm{h}^{-1}\right)$ & $1534 \pm 234$ & $1521 \pm 242$ & $1546 \pm 233$ \\
\hline Moderate-intensity running $\left(>8.0\right.$ to $\left.13.0 \mathrm{~km} \cdot \mathrm{h}^{-1}\right)$ & $648 \pm 146$ & $659 \pm 133$ & $637 \pm 161$ \\
\hline High-intensity running $\left(>13.0\right.$ to $\left.18.0 \mathrm{~km} \cdot \mathrm{h}^{-1}\right)$ & $160 \pm 76$ & $172 \pm 58$ & $148 \pm 86$ \\
\hline Sprinting $\left(>18.0 \mathrm{~km} \cdot \mathrm{h}^{-1}\right)$ & $22 \pm 20$ & $29 \pm 21$ & $16 \pm 16$ \\
\hline
\end{tabular}

Abbreviations: GPS $=$ Global Positioning System. MSFT $=$ Multi-Stage Fitness Test ${ }^{\mathrm{b}}$ Relative to the maximum heart rate attained during the MSFT

* Difference between high- and low-fit groups, $p<.01$ 
$\left(t_{(32)}=2.7, p=0.010\right)$ heart rate, as well as relative exercise intensity $\left(t_{(32)}=-4.1, p<0.001\right)$, were lower in the high-fit group compared to the low-fit group (Table 2). Average heart rate across the whole $5 \mathrm{~h}$ exercise trial $\left(105 \pm 13\right.$ beats $\left.\mathrm{min}^{-1}\right)$ was higher than during the whole $5 \mathrm{~h}$ resting trial $(84 \pm 11$ beats $\min ^{-1}, t_{(35)}=11.8, p<0.001$ ).

During the football session, the average total distance covered by the group overall was $2788 \pm 432 \mathrm{~m}$, with the low-fit participants covering a total of $2792 \pm 474 \mathrm{~m}$ and high-fit participants covering a total of $2786 \pm 402 \mathrm{~m}$. There was no significant effect of fitness on the total distance and distance covered across the six different speed categories (Pillai's trace: $p=0.301$, Table 2).

\subsection{Metabolic responses}

Blood glucose and plasma insulin concentration across the exercise and resting trial, for the group overall as well as the high- and low-fit group, can be seen in Table 3.

\subsubsection{Blood glucose concentration}

3.2.1.1. Response to exercise. Blood glucose concentration was similar between the exercise and resting trials (main effect of trial; $p=0.063$ ), but changed over time (main effect of time; $\left.F_{(2,77)}=29.5, p<0.001\right)$. This pattern of change was different between the exercise and resting trial (trial by time interaction; $F_{(2,81)}=11.6, p<0.001$, Figure 2). Specifically, blood glucose concentration was lower $60 \mathrm{~min}$ post-exercise compared to 60 min post-rest $\left(t_{(35)}=-6.3, p<0.001, \mathrm{MD}=-0.80 \mathrm{mmol} \cdot \mathrm{L}^{-1}\right.$, $95 \% \mathrm{Cl}\left[-1.06,-0.55 \mathrm{mmol} \cdot \mathrm{L}^{-1}\right]$, Table 3). Blood glucose concentration was similar between high- and low-fit groups (main effect of fitness; $p=0.596$ ), and fitness did not moderate the pattern of change (trial by time by fitness interaction; $p=0.234)$.

\subsubsection{Postprandial response to standardised lunch.}

Following the standardised lunch, blood glucose concentration was lower during the exercise trial compared to the resting trial (main effect of trial; $F_{(1,34)}=15.2, p<0.001$, $\left.\mathrm{MD}=-0.25 \mathrm{mmol} \cdot \mathrm{L}^{-1}, 95 \% \mathrm{Cl}\left[-0.37,-0.12 \mathrm{mmol} \cdot \mathrm{L}^{-1}\right]\right)$ as well as changing over time (main effect of time; $F_{(1,102)}=56.8$, $p<0.001$ ). The pattern of change was different between the exercise and resting trials (trial by time interaction; $F_{(3,102)}=10.4$, $p<0.001$ ), whereby blood glucose concentration was lower before lunch during the exercise trial compared to the control trial (Table 3). Blood glucose concentration following lunch was similar between the high- and low-fit groups (main effect of fitness; $p=0.373$ ), and fitness did not moderate the pattern of change (trial by time by fitness interaction; $p=0.368$ ).

Blood glucose tAUC following the standardised lunch was similar between the exercise and resting trial $(p=0.124)$, as well as between the high- and low-fit groups (main effect of fitness; $p=0.551$ ). The difference between trials was similar for the high- and low-fit groups (trial*fitness interaction; $p=0.177$ ).

3.2.1.3. Fasted day 1 and day 2 measures. Overall fasted blood glucose concentration was similar between the exercise and resting trials (main effect of trial; $p=0.812$ ) and over time (main effect of time; $p=0.071$ ). The pattern of change between

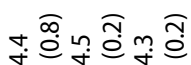

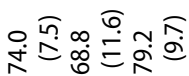

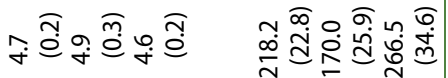

Fั

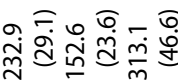

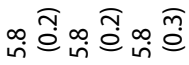

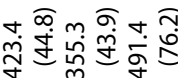

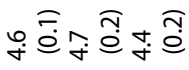

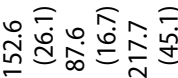

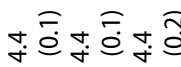

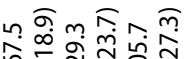

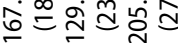

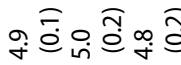

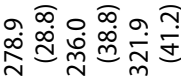

m

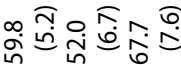

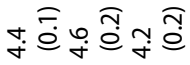

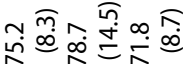

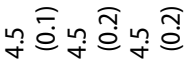

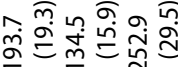

そุ

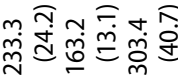

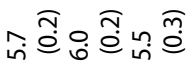

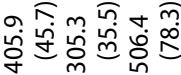

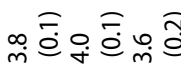

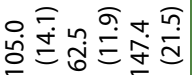

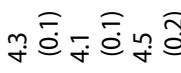

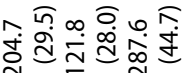

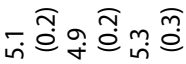

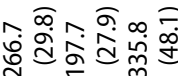

ๆ

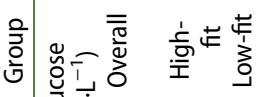

宅高

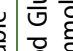


the exercise and resting trial was similar (trial by time interaction; $p=0.663$ ). Fasted blood glucose concentration was similar between the high- and low-fit group (main effect of fitness; $p=0.175$ ) and fitness did not moderate the pattern of change (trial by time by fitness interaction; $p=0.090$ ).

\subsubsection{Plasma insulin concentration}

3.2.2.1. Response to exercise. Plasma insulin concentration was similar between the exercise and resting trial (main effect of trial; $p=0.766$ ), but changed over time (main effect of time; $\left.F_{(2,80)}=59.1, p<0.001\right)$. The pattern of change was similar between the exercise and resting trials (trial by time interaction; $p=0.089$, Figure 2). Plasma insulin concentration was lower in the high-fit group compared to the low-fit group (main effect of fitness; $F_{(1,34)}=10.9, p=0.002, \mathrm{MD}=-88.4 \mathrm{pmol} \cdot \mathrm{L}^{-1}, 95 \% \mathrm{Cl}$ $\left.\left[-142.6,-34.1 \mathrm{pmol} \cdot \mathrm{L}^{-1}\right]\right)$. Fitness did not moderate the pattern of change (trial by time by fitness interaction; $p=0.186$ ).

\subsubsection{Postprandial response to standardised lunch.}

Following the standardised lunch, overall plasma insulin concentration was similar between the exercise and resting trials (main effect of trial; $p=0.198$ ) but changed over time (main effect of time; $\left.F_{(1,102)}=46.5, p<0.001\right)$. The pattern of change was similar between the exercise and resting trials (trial by time interaction; $p=0.544$ ). Plasma insulin concentration was lower in the high-fit group compared to the low-fit group (main effect of fitness; $F_{(1,34)}=11.5, p=0.002, \mathrm{MD}=-133.5 \mathrm{pmol} \cdot \mathrm{L}^{-1}, 95 \% \mathrm{Cl}$ $\left.\left[-213.5,-53.5 \mathrm{pmol} \cdot \mathrm{L}^{-1}\right]\right)$. Fitness did not moderate the pattern of change in response to lunch (trial by time by fitness interaction; $p=0.368$ ).

Plasma insulin tAUC following the standardised lunch was similar between the exercise and resting trial (main effect of trial; $p=0.207$ ), but was lower in the high-fit group compared to the low-fit group (main effect of fitness; $F_{(1,34)}=10.9$, $p=0.002, \mathrm{MD}=-2672.6 \mathrm{pmol} \cdot \mathrm{L}^{-1} \times 120 \mathrm{~min}, 95 \% \mathrm{Cl}$ [-4321.1, -1024.0 pmol.t $\left.{ }^{-1} \times 120 \mathrm{~min}\right]$; Figure 3). However, fitness did not moderate plasma insulin tAUC (trial by fitness interaction; $p=0.952$ ).

3.2.2.3. Fasted day 1 and day 2 measures. Overall plasma insulin concentration across fasted samples on day 1 and day 2 was similar between the exercise and resting trials (main effect of trial; $p=0.446$ ), and between day 1 and day 2 (main effect of time; $p=0.181$ ). The pattern of change was similar between the exercise and resting trial (trial by time interaction; $p=0.604$ ). Fasted plasma insulin concentration was similar between the high- and low-fit groups (main effect of fitness; $p=0.496$ ), with no moderating effect of fitness on the pattern of change (trial by time by fitness interaction; $p=0.568$ ).

\section{Discussion}

The main findings of the present study were that the postprandial insulinaemic response to an ecologically valid meal was $70 \%$ lower in the high-fit than in the low-fit participants, demonstrating that higher levels of physical fitness are associated with enhanced metabolic health in adolescents. In addition, whilst postprandial glycaemia and insulinaemia were

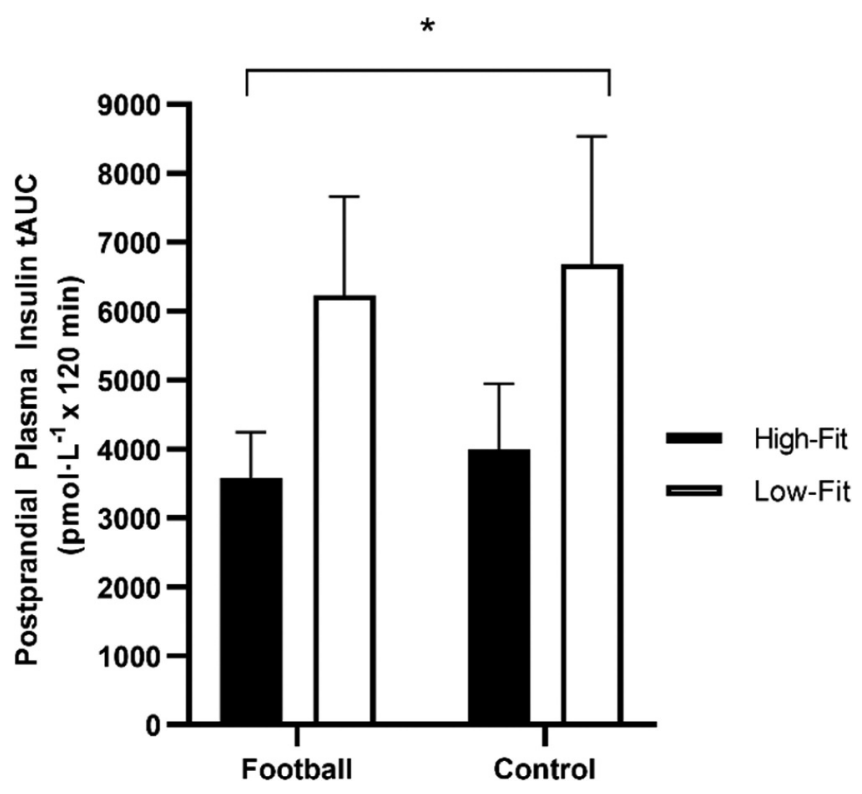

Figure 3. Postprandial plasma insulin tAUC following the football and control trials, for the high- (filled bars) and low-fit groups (open bars). Data are mean \pm $95 \% \mathrm{Cl}$. *main effect of fitness, $p<0.001$.
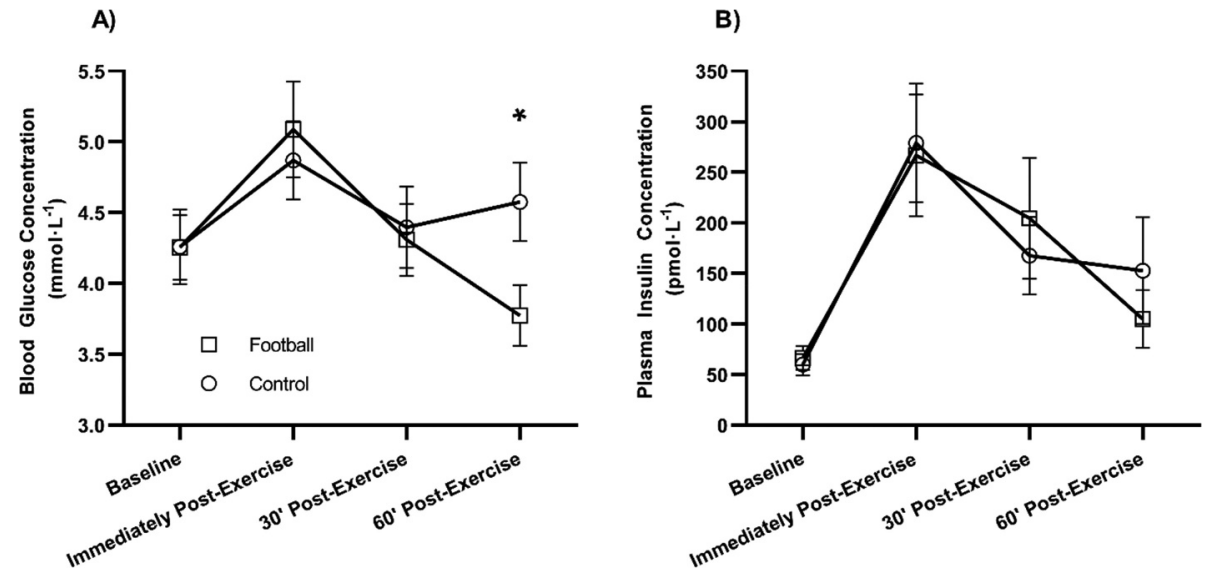

Figure 2. Overall post-exercise blood glucose (A) and plasma insulin (B) concentrations for the football and control trials. Data are mean $\pm 95 \%$ Cl. ${ }^{*}$ difference between football and control, $p<0.001$. 
unaffected by a 60 min bout of football activity, blood glucose concentration was $21 \%$ lower $60 \mathrm{~min}$ post-exercise.

The present study is the first to provide evidence demonstrating that the postprandial insulinaemic response was, on average, $70 \%$ lower in those considered high-fit compared to their low-fit counterparts. This is an important finding, given the role that postprandial insulinaemia plays in the development of insulin resistance and cardiovascular risk (DiNicolantonio et al., 2017; Lautt, 2007). A difference of such magnitude emphasises the crucial role of physical fitness in adolescents for enhancing lifelong health. One possible explanation for this strong effect of fitness might be due to the increased GLUT4 translocation and skeletal muscle capillarisation, which is suggested to be a result of chronic exercise training (Bird \& Hawley, 2017). A further possible explanation could be attributed to the body composition of participants, given that body composition is considered a risk factor for insulin resistance (Arslanian and Suprasongsin, 1996; Arslanian, 2000). Unsurprisingly, the characteristics relating to body composition were more favourable in the high-fit group compared to the low-fit group (Table 1). A recent study supports these findings by showing that adolescents considered overweight/obese had a higher postprandial insulinaemic response by $\sim 36 \%$ (Short et al., 2018). However, $\dot{\mathrm{V}}_{2}$ peak in the aforementioned study (Short et al., 2018) was normalised to lean body mass, rather than total body mass, which makes comparisons with the characteristics in the current study difficult. Whilst the cross-sectional nature of the relationship between physical fitness and metabolic health in the present study prevents the inference of a causal relationship, the present study provides important novel findings that those with a higher physical fitness - non-invasively measured via the MSFT - have a lower postprandial insulinaemic response.

The present study provides novel information regarding the postprandial insulinaemic response to an ecologically valid meal following an acute bout of football; with the current data suggesting that $60 \mathrm{~min}$ football activity does not affect postprandial insulinaemic responses. Conversely, previous studies have demonstrated acute reductions in the postprandial insulinaemic response to a test meal (Short et al., 2018, 2013) and an OGTT (Cockcroft et al., 2015, 2017) following highintensity intermittent cycling exercise (Cockcroft et al., 2015, 2017) and aerobic circuit exercise (Short et al., 2018, 2013). Possible explanations for divergent results might reside in the fact that previous studies were conducted in a tightly controlled laboratory environment, used an OGTT as the test meal (Cockcroft et al., 2015, 2017), recruited participants with habitually low physical activity (Short et al., 2013) or were overweight/obese (Short et al., 2018). A previous study has shown that an acute bout of basketball reduces postprandial insulinaemia (by $35 \%$ ) following a standardised mixed-meal (Dring et al., 2019a), in comparison with a non-statistically significant reduction of $11 \%$ in the present study. The present study and the study by Dring et al. (2019a) are the only ones, to the authors' knowledge, that examine postprandial insulinaemia in response to games-based activity. Although the exercise bouts were of similar duration and relative exercise intensity, in the basketball study (Dring et al., 2019a) the participants had a higher predicted $\mathrm{VO}_{2}$ peak than the participants in the present study. Therefore, the absolute exercise intensity may have been higher in the basketball study (Dring et al., 2019a), accounting for the greater reduction in postprandial insulinaemia ( $35 \%$ vs. $11 \%$ in the present study).

The present study also provides novel information regarding the transient reduction (21\%) in blood glucose 60 min following an acute bout of football in adolescents. This is of greater magnitude compared to the $11 \%$ reduction following an acute bout of basketball (Dring et al., 2019a), despite similar duration and relative exercise intensities seen between the studies. A possible explanation for this may be differences in the movement patterns, muscle fibre recruitment, and active muscle mass, between the two activities; though these suggestions remain speculative at this stage. However, data from the current study and previous work (Dring et al., 2019a) suggest that gamesbased activity does not affect the postprandial glycaemic response to a standardised meal. This conflicts with previous work using football (Smallcombe et al., 2018) and circuit-based exercise (Short et al., 2018, 2013); although participants were recruited based on low habitual physical activity in previous work (Short et al., 2013), which may explain these discrepancies. The findings of the present study do however have important ecological application, as acute bouts of games-based exercise incorporated into the daily school routines of adolescents could enhance glucose regulation. Future work should continue to examine the underlying factors that determine the acute glycaemic and insulinaemic responses to different physical activities, particularly with regards to the modality, movement patterns and intensity of the activity.

The variance in physical fitness levels between study participants has been cited as a potential factor which may explain why exercise effects are seen in some previous studies (Cockcroft et al., 2015; Dring et al., 2019a). These suggestions have been made when comparing across separate studies, with no empirical research to date investigating if physical fitness may moderate the response to exercise. The results of the current study provide novel evidence that there was no moderating effect of physical fitness on the postprandial glycaemic and insulinaemic responses, even though fitness was positively related to the overall postprandial insulinaemic response. Despite the metabolic responses being similar between the high- and low-fit groups, the relative exercise intensity of the football session was higher in the low-fit group ( $80 \%$ HRmax) compared to the high-fit group (70\% HRmax). Furthermore, the external load - assessed by GPS - was similar between the fitness groups (Table 2). This suggests that, in the present study, even though low-fit participants exercised at a higher relative intensity for a similar external load, there was no additional metabolic benefit. Indeed, this is a novel contribution to the literature and future studies should seek to replicate this finding, whilst also increasing the exercise intensity.

The skill level and football experience of the participants was not assessed in the present study, which may be a limitation. Given that there is the potential for all young people to participate in football (or indeed any games-based activity) at school, we did not want to exclude participants based upon their football history or skill level. We also decided to use mixed groups of boys and girls, again because this is often how sessions are run in schools. However, future studies could 
consider whether factors that may affect the activity patterns of games-based activity (such as skill level and sex) also have a resultant effect upon the potential health benefits.

In summary, although a 60 min bout of football did not affect the postprandial insulinaemic response, the exercise led to a transient reduction in blood glucose concentration $60 \mathrm{~min}$ post-exercise, irrespective of physical fitness. This is an important finding, as football is the most popular team sport played by adolescents in England. This highlights the ecological validity of such a modality, with potential to improve adherence in this population and thus, improving glucose regulation. Moreover, the present study provides novel evidence that physical fitness is important for the postprandial insulinaemic response to a standardised meal, with a $\sim 70 \%$ lower postprandial insulinaemic response in high-fit adolescents compared to their low-fit counterparts. This further strengthens the argument for promoting improved physical fitness amongst school-aged children, to help combat the development of risk factors for cardiometabolic disease and enhance lifelong health.

\section{Disclosure statement}

No potential conflict of interest was reported by the authors.

\section{ORCID}

Ryan A. Williams (D) http://orcid.org/0000-0002-1346-7756 Simon Cooper (ID) http://orcid.org/0000-0001-5219-5020 Karah J. Dring (ID) http://orcid.org/0000-0002-9647-3579 John G. Morris (iD) http://orcid.org/0000-0001-6508-7897 Caroline Sunderland (D) http://orcid.org/0000-0001-7484-1345 Mary E. Nevill (D) http://orcid.org/0000-0003-2498-9493

\section{Data availability}

Data are available from the corresponding author upon reasonable request.

\section{References}

Arslanian, S. A. (2000, January 1). Type 2 diabetes mellitus in children: Pathophysiology and risk factors. Journal of Pediatric Endocrinology and Metabolism, [Internet], 13, 1385 - 1394. (Supplement). Retrieved July 23, 2020, from https://www.degruyter.com/doi/10.1515/jpem-2000-s612

Arslanian, S. A., \& Suprasongsin, C. (1996). insulin sensitivity, lipids and body composition in childhood: Is 'Syndrome $X$ ' present? The Journal of Clinical Endocrinology \& Metabolism, 81(3), 1058-1062. https://doi.org/ 10.1210/jcem.81.3.8772576

Artero, E. G., España-Romero, V., Castro-Piñero, J., Ortega, F. B., Suni, J., Castillo-Garzon, M. J., \& Ruiz, J. R. (2011, March). Reliability of field-based fitness tests in youth. International Journal of Sports Medicine, 32(3), 159-169. https://doi.org/10.1055/s-0030-1268488

Bailey, R. C., Olson, J., Pepper, S. L., Porszasz, J., Barstow, T. J., \& Cooper, D. M. (1995, July). The level and tempo of children's physical activities: An observational study. Medicine \& Science in Sports \& Exercise, 27(7), 1033-1041. https://doi.org/10.1249/00005768-199507000-00012

Barnett, A., Chan, L. Y. S., \& Bruce, L. C. (1993, February). A preliminary study of the 20-m multistage shuttle run as a predictor of peak VO2 in Hong Kong Chinese students. Pediatric Exercise Science, 5(1), 42-50. https://doi.org/10.1123/pes.5.1.42

Batacan, R. B., Duncan, M. J., Dalbo, V. J., Tucker, P. S., \& Fenning, A. S. (2017, March). Effects of high-intensity interval training on cardiometabolic health: A systematic review and meta-analysis of intervention studies.
British Journal of Sports Medicine, 51(6), 494-503. https://doi.org/10. 1136/bjsports-2015-095841

Bird, S. R., \& Hawley, J. A. (2017, March). Update on the effects of physical activity on insulin sensitivity in humans. BMJ Open Sport \& Exercise Medicine, 2(1), e000143. https://doi.org/10.1136/bmjsem-2016-000143

Bond, B., Weston, K. L., Williams, C. A., \& Barker, A. R. (2017). Perspectives on high-intensity interval exercise for health promotion in children and adolescents. Journal of Sports Medicine, 27(8), 243-265. https://doi.org/ 10.2147/OAJSM.S127395

Booth, F. W., Roberts, C. K., \& Laye, M. J. (2012, April). Lack of exercise is a major cause of chronic diseases. Comprehensive Physiology, 2(2), 1143-1211. https://doi.org/10.1002/cphy.c110025

Cockcroft, E. J., Williams, C. A., Tomlinson, O. W., Vlachopoulos, D., Jackman, S. R., Armstrong, N., \& Barker, A. R. (2015, November). High intensity interval exercise is an effective alternative to moderate intensity exercise for improving glucose tolerance and insulin sensitivity in adolescent boys. Journal of Science and Medicine in Sport, 18(6), 720-724. https://doi.org/10.1016/j.jsams.2014.10.001

Cockcroft, E. J., Williams, C. A., Weaver, H., O'Connor, A., Jackman, S. R., Armstrong, N., \& Barker, A. (2017, November). Acute exercise and insulin sensitivity in boys: A time-course study. International Journal of Sports Medicine, 38(13), 967-974. https://doi.org/10.1055/s-0043-118007

Cooper, S. B., Bandelow, S., Nute, M. L., Dring, K. J., Stannard, R. L., Morris, J. G., \& Nevill, M. E. (2016, June). Sprint-based exercise and cognitive function in adolescents. Preventive Medicine Reports, 7(4), 155-161. https://doi.org/10.1016/j.pmedr.2016.06.004

DiNicolantonio, J. J., Bhutani, J., OKeefe, J. H., \& Crofts, C. (2017). Postprandial insulin assay as the earliest biomarker for diagnosing pre-diabetes, type 2 diabetes and increased cardiovascular risk. Open Heart, 4(2), e000656. https://doi.org/10.1136/openhrt-2017-000656

Dring, K. J., Cooper, S. B., Morris, J. G., Sunderland, C., Foulds, G. A., Pockley, A. G., \& Nevill, M. E. (2019a, April). Cytokine, glycemic, and insulinemic responses to an acute bout of games-based activity in adolescents. Scandinavian Journal of Medicine \& Science in Sports, 29(4), 597-605. https://doi.org/10.1111/sms.13378

Dring, K. J., Cooper, S. B., Morris, J. G., Sunderland, C., Foulds, G. A., Pockley, A. G., \& Nevill, M. E. (2019b). Multi-stage fitness test performance, VंO2 peak and adiposity: Effect on risk factors for cardiometabolic disease in adolescents. Frontiers in Physiology, 10, 629. https://doi.org/10.3389/fphys.2019.00629

Ekelund, U., Anderssen, S. A., Froberg, K., Sardinha, L. B., Andersen, L. B., \& Brage, S. (2007, August). Independent associations of physical activity and cardiorespiratory fitness with metabolic risk factors in children: The European youth heart study. Diabetologia, 50(9), 1832-1840. https://doi. org/10.1007/s00125-007-0762-5

Ekelund, U., Luan, J., Sherar, L. B., Esliger, D. W., Griew, P., \& Cooper, A. (2012, February 15). Moderate to vigorous physical activity and sedentary time and cardiometabolic risk factors in children and adolescents. JAMA, 307 (7), 704-712. https://doi.org/10.1001/jama.2012.156

Gleeson, M., Bishop, N. C., Stensel, D. J., Lindley, M. R., Mastana, S. S., \& Nimmo, M. A. $(2011,5)$. The anti-inflammatory effects of exercise: Mechanisms and implications for the prevention and treatment of disease. Nature Reviews Immunology, 11(9), 607-615. https://doi.org/10. 1038/nri3041

Haapala, E. A., Wiklund, P., Lintu, N., Tompuri, T., Väistö, J., Finni, T., TARKKA, I. M., KEMPPAINEN, T., BARKER, A. R., EKELUND, U., BRAGE, S., \& LAKKA, T. A. (2020, May). Cardiorespiratory fitness, physical activity, and insulin resistance in children. Medicine \& Science in Sports \& Exercise, 52(5), 1144-1152. https://doi.org/10.1249/MSS.0000000000002216

Howe, C. A., Freedson, P. S., Feldman, H. A., \& Osganian, S. K. (2010, December). Energy expenditure and enjoyment of common children's games in a simulated free-play environment. The Journal of Pediatrics, 157(6), 936-942.e1-2. https://doi.org/10.1016/j.jpeds.2010.06.041

Krustrup, P., \& Bangsbo, J. (2015, November). Recreational football is effective in the treatment of non-communicable diseases. British Journal of Sports Medicine, 49(22), 1426-1427. https://doi.org/10.1136/bjsports2015-094955

Lautt, W. W. (2007, Oct). Postprandial insulin resistance as an early predictor of cardiovascular risk. Therapeutics and Clinical Risk Management, 3(5), 761-770. https://www.ncbi.nlm.nih.gov/pmc/articles/PMC2376071/ 
Mendham, A. E., Coutts, A. J., \& Duffield, R. (2012, November). The acute effects of aerobic exercise and modified rugby on inflammation and glucose homeostasis within Indigenous Australians. European Journal of Applied Physiology, 112(11), 3787-3795. https://doi.org/10.1007/s00421012-2361-5

Mendham, A. E., Duffield, R., Marino, F., \& Coutts, A. J. (2015, November). Differences in the acute inflammatory and glucose regulatory responses between small-sided games and cycling in sedentary, middle-aged men. Journal of Science and Medicine in Sport, 18(6), 714-719. https://doi.org/ 10.1016/j.jsams.2014.09.008

Moore, S. A., McKay, H. A., Macdonald, H., Nettlefold, L., Baxter-Jones, A. D. G., Cameron, N., \& BRASHER, P. M. A. (2015, August). Enhancing a somatic maturity prediction model. Medicine \& Science in Sports \& Exercise, 47(8), 1755-1764. https://doi.org/10.1249/MSS. 0000000000000588

Ramsbottom, R., Brewer, J., \& Williams, C. (1988, December). A progressive shuttle run test to estimate maximal oxygen uptake. British Journal of Sports Medicine, 22(4), 141-144. https://doi.org/10. 1136/bjsm.22.4.141

Reaven, G. (2005, November). Insulin resistance, type 2 diabetes mellitus, and cardiovascular disease: The end of the beginning. Circulation, 112 (20), 3030-3032. https://doi.org/10.1161/CIRCULATIONAHA.105.504670

Reinehr, T. (2013, December). Type 2 diabetes mellitus in children and adolescents. World Journal of Diabetes, 4(6), 270-281. https://doi.org/ 10.4239/wjd.v4.i6.270

Röhling, M., Herder, C., Stemper, T., \& Müssig, K. (2016). Influence of acute and chronic exercise on glucose uptake. Journal of Diabetes Research, 2016, 2868652. https://doi.org/10.1155/2016/2868652

Scholes, S., \& Mindell, J. (2016). Health survey for England 2015 physical activity in children. Health Social Care Interface Center. http://healthsurvey.hscic.gov.uk/media/37752/hse2015-child-phy-act.pdf

Short, K. R., Pratt, L. V., \& Teague, A. M. (2018, April). A single exercise session increases insulin sensitivity in normal weight and overweight/obese adolescents. Pediatric Diabetes, 19(6), 1050-1057. https://doi.org/10. 1111/pedi.12684

Short, K. R., Pratt, L. V., Teague, A. M., Man, C. D., \& Cobelli, C. (2013, March). Postprandial improvement in insulin sensitivity after a single exercise session in adolescents with low aerobic fitness and physical activity.
Pediatric Diabetes, 14(2), 129-137. https://doi.org/10.1111/j.1399-5448. 2012.00900.x

Smallcombe, J. W., Barrett, L. A., Morris, J. G., Sherar, L. B., \& Tolfrey, K. (2018). Small-sided soccer in school reduces postprandial lipemia in adolescent boys. Medicine \& Science in Sports \& Exercise, 50(11), 2351-2359. https:// doi.org/10.1249/MSS.0000000000001702

Svensson, M., \& Drust, B. (2005, June). Testing soccer players. Journal of Sports Sciences, 23(6), 601-618. https://doi.org/10.1080/ 02640410400021294

Tagi, V. M., Giannini, C., \& Chiarelli, F. (2019, June 4). Insulin resistance in children. Front Endocrinology, 10(342). Retrieved July 23, 2020, from https://www.ncbi.nlm.nih.gov/pmc/articles/PMC6558106/

Taking Part 2017/18: annual child release [Internet]. Department for digital, culture, media \& sport. GOV.UK. Retrieved July 23, 2020, from], https:// www.gov.uk/government/statistics/taking-part-201718-annual-childrelease

Tomkinson, G. R., Lang, J. J., Blanchard, J., Léger, L. A., \& Tremblay, M. S. (2019, May). The 20-m shuttle run: Assessment and interpretation of data in relation to youth aerobic fitness and health. Pediatric Exercise Science, 31(2), 152-163. https://doi.org/10.1123/pes.2018-0179

Townsend, N., Wickramasinghe, K., Williams, J., \& Bhatnagar, P. (2015). Physical activity statistics 2015. Br Heart Found. https://www.bhf.org. uk/informationsupport/publications/statistics/physical-activity-statistics-2015

Tuomilehto, J., Lindström, J., Eriksson, J. G., Valle, T. T., Hämäläinen, H., IlanneParikka, P., Keinänen-Kiukaanniemi, S., Laakso, M., Louheranta, A., Rastas, M., Salminen, V., Aunola, S., Cepaitis, Z., Moltchanov, V., Hakumäki, M., Mannelin, M., Martikkala, V., Sundvall, J., \& Uusitupa, M. (2001, May). Prevention of type 2 diabetes mellitus by changes in lifestyle among subjects with impaired glucose tolerance. New England Journal of Medicine, 344(18), 1343-1350. https://doi.org/10.1056/ NEJM200105033441801

Wolever, T. M., Jenkins, D. J., Jenkins, A. L., \& Josse, R. G. (1991, November). The glycemic index: Methodology and clinical implications. The American Journal of Clinical Nutrition, 54(5), 846-854. https://doi.org/ 10.1093/ajcn/54.5.846

World Health Organisation. (2008). Waist circumference and waist-hip ratio. Geneva: World Health Organisation. 